high current, but may be made with a low current, if formic acid is the electrolyte.

This study is, of course, only a fragnent of what remains to be done.

UTiversity of PENNSYlvania,

Philadelpita, PA.

\title{
INVESTIGATION OF THE CLAISEN CONDENSATION. III. FURTHER CONTRIBUTIONS TOWARDS THE ELUCIDATION OF THE MECHANISM OF THE REACTION. ${ }^{1}$
}

BY J. Bishop Tingie And ERnest H. Gorsline.

Received October 7, 1908.

The various current theories regarding the mechanism of the ethyl acetoacetate condensation were discussed in our second paper. It is, therefore, only necessary in the present communication to state that, broadly, the question is whether the sodium, or other condensing agent, acts directly on the ethyl acetate, or whether it reacts first with a trace of alcohol, the actual condensation being brought about by the sodium ethylate so produced. It is evident that valuable evidence on the question at issue night be obtained by an examination of the behavior of ethyl acetate free from alcohol. Accordingly, our early efforts were directed towards the purification of this ester. The product which we eventually obtained was probably the purest specimen of this compound hitherto prepared. It reacted with sodium, to form ethyl acetoacetate, as readily as the ordinary "pure" material.

We think that this effectively disposes of the idea that the action is caused by the presence of a trace of alcohol in the ester, unless, of course, the statement be made that ethyl acetate is aliagy dissociated to some infinitesimal extent into alcohol, or into some hypothetical "ethylidene," or "active variety of ethylene," whatever these may be. Statements of this nature, though they are made not infrequently, are outside discussion, because they assume the very point at issue.

Much work has been clone by A. Michael, Nef and others on the purification of ethyl acetate and also on the quantity of hydrogen evolved from sodium and ethyl acetate. This quantity is not equivalent to that of the reacting sodium. A portion of the gas always attacks certain of the other substances which are present.

We have carried ont a very large number of experiments with the object of eliminating as far as possible the effect of this absorbed gas. We added to the reacting nuterials a number of easily reducible substances in the hope that the hydrogen would attack them in preference to reacting with the ethyl acetate derivatives. Our results were not very encouraging.

${ }^{1}$ Our previous papers of this series appeared in the Am. Chem. J., 27,483 (1907); 40, 46 (1908). 
In continuation of our work on the action of sodium on esters, ${ }^{1}$ we have confirmed our observation that ethyl malonate reacts with two atomic proportions of sodium, hydrogen being evolved only during the earlier part of the experiment, when the first half of the metal is reacting. Ethyl dimethyl malonate and ethyl chlormalonate react with four and one atomic proportions of sodium, respectively. These results appear to prove that diethyl malonate has a different structure from the other two esters. As mentioned in our earlier paper, its formula is probably, $\mathrm{C}_{2} \mathrm{H}_{5} \mathrm{OCOCH}: \mathrm{C}(\mathrm{OH}) \mathrm{OC}_{2} \mathrm{H}_{5}$. The monosodium derivative is, doubtless, $\mathrm{C}_{2} \mathrm{H}_{5} \mathrm{OCOCH}: \mathrm{C}(\mathrm{ONa}) \mathrm{OC}_{2} \mathrm{H}_{5}$, and, from analogy with ethyl benzoate, it is most likely that the second atom of metal attacks the ethoxy group giving $\mathrm{C}_{2} \mathrm{H}_{5} \mathrm{OCOCH}: \mathrm{C}(\mathrm{ONa}) \mathrm{C}(\mathrm{ONa}): \mathrm{CHCO}_{2} \mathrm{C}_{2} \mathrm{H}_{5}$, although, of course, the carbethoxy group may be involved, and the two complexes which result may be linked together by the $C O$ groups. We hope to offer some evidence on this point later. Ethyl dimethylmalonate behaves, mutatis mutandis, like ethyl benzoate, and, therefore, the sodium derivative must be formulated as $\mathrm{Me}_{2} \mathrm{C}<{ }_{\mathrm{CONa}}^{\mathrm{CONa}}$. The possibility that it may have the double formula is sufficiently obvious. The original ester agrees in behavior with the current formula, $\left(\mathrm{CH}_{3}\right)_{2} \mathrm{C}\left(\mathrm{CO}_{2} \mathrm{C}_{2} \mathrm{H}\right)_{2}$. Ethyl chlormalonate acts as though a limited portion of it were in the hydroxy form; more work will be required before a satisfactory explanation of its behavior can be given.

Since the publication of our earlier paper A. Wahl has communicated some very interesting results obtained by the study of the action of sodium on a variety of esters. ${ }^{2}$

In the light of our knowledge that ether and tertiary bases act catalytically in promoting the Claisen condensation we have investigated the more important reactions which Claisen studied. We made parallel experiments, in the one series excluding ether, whereas, in the other it was added to the reacting substances. In each case it was found that the compounds react with such great rapidity that it was not possible to observe any acceleration caused by the ether. It is, doubtless, for this reason that Claisen himself failed to observe the catalyzing influence of this substance. Included in the same section we give a brief description of some additional work on the condensation of camphor with certain esters. The results demonstrate that the method of work and the conditions employed may require considerable variation for different esters. This is particularly true, for example, with ethyl benzoate and ethyl phthalate.

\footnotetext{
1 Am. Chem. J., 40, 46 (Ig08).

2 Compt. rend., I47, 72 .
} 
We also describe the preparation of a condensation compound from benzophenone and ethyl oxalate. It was obtained as an oil and has not yet been studied. It gives, in alcoholic solution, an intense coloration with an aqueous solution of ferric chloride. We desire to reserve the study of this interesting compound from benzophenone. After the conclusion of our work we noticed the publication of a paper by $W$. Iiecknant and $A$. Kron' in which they also showed that tertiary ketones, R CHCO, may undergo the Claisen condensation. This fact is entirely incompatible with the Claisen-Nef theory of the reaction, whereas it might well be anticipated from A. Michael's explanation of the mechanism of the condensation.

At the end of the present paper we sive a brief record of a few experiments on the interaction of sodium and benzophenone. ${ }^{2}$ The product consists of a deep blue powder which yields a mixture of benzhydrol and benzpinacone when treated with water. A colorless crystallinc derivative is obtained by the action of metanitrobenzoyl chloride on the blue powder.

We made numerous experiments, under varying conditions, with the object of condensing acetaldehyde with ethyl oxalate, ethyl formate and camphor, respectively. In no case were we successful because the sodium, or more probably some sodium compound, caused the aldehyde to polymerize with very great rapidity. We describe one of the experiments only, because it led to the production of a rather curious substance.

\section{Experimental.}

I. Experiments wim Iimy Acriate--Kahlbaum's best ethyl acetate, which was used in all our experiments, was dried over calcium chloride. A little sodium wire was now added, the liquid shaken for a few moments, then filtered quickly and redistilled. The product was divided into two portions, each of which was treated with sodium ( 1 atom : 3 mol. of ester) in the usual manner, but to one portion 25 drops of absolute ether were added. The reaction was complete after two hours at the room temperature. The yield of ethyl acetoacetate appeared to be distinctly larger from the experiment in which ether was used.

In a second scrics of experiments the ethyl acetate was shaken with fused sodium acetate and then distilled from it. The distillate was shaken first with calcinn chloride solution, then with water. It was now dried over calcium chloride anci distilled. It boiled at $77^{\circ}$ and failed to react with litmus paper.

Thiml Serics.-.The product from the second series of experiments was allowed to remain over phosphoric anhydride during 24 hours, then boiled with it for two hours, and finally distilled off. It boiled at $77^{\circ}$ and had

${ }^{1} \mathrm{Bcr}, 4 \mathrm{I}, \mathrm{I} 260$ (1908).

${ }^{2}$ Acree: Am. Chem. J., 29,604 (1903). 
an acid reaction; the anhydride became dark. The distillate was neutralized with a dilute aqueous solution of sodium carbonate, washed with water, dried over calcium chloride and filtered.

Fourth Series. - The product from the third series of experiments was boiled in contact with phosphoric anhydride for three hours and then distilled. It boiled at $77^{\circ}$ and was neutral to litmus. The ester from the second, third and fourth series, when treated with sodium, behaves like that obtained by the first method of purification.

Fifth Series.--The ester as obtained from Kahlbaum was dried over calcium chloride, distilled, and then boiled during three working days with calcium turnings, which remained entirely untarnished. The ester was then distilled.

Sixth Series.--Isster from the fourth series was treated as in the fifth series. The ester from the fifth and sixth series reacted quickly with sodium, the experiments and results being similar to those described in connection with the first series. In order to show how sensitive calcium is to alcohol, some of the metallic turnings were covered with absolute alcohol (Kahlbaum) in a flask protected from moisture. Reaction soon commenced without heating and after 3 days all the metal had disappeared.

In order to see if the state of division of the sodium had any material influence on the results, some of the ester from the fifth series was treated with sodium chips instead of wire. The action was slower, but it was complete after three hours at the room temperature and three hours' boiling.

Duplicates were made of all the condensation experiments described above, except that the ratio of ester to sodium was 2 mol. : I atom. The results were entirely similar.

II. Synthesis of Ethyl Acetoachtate usin; Fithis AS Solvent.Ethyl acetate (1 7.6 grams, 2 mols.), sodium (2.3 grams, I atom) and absolute ether $(25 \mathrm{cc}$.), when mixed, reacted vigorously. After being boiled for five hours the reaction was complete. The product was a dark brown liquid, but after standing over-night it became a red solid. It was acidified with dilute acetic acid ( $I: I)$, poured into saturated brine and extracted four times with ether. After being dried, the ether was distilled, the temperature being raised to $95^{\circ}$. The residue boiled at $100^{\circ}$ ( $\mathrm{I}$ I $\mathrm{mm}$.)

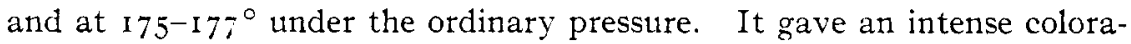
tion with aqueous solution of ferric chloride and is, doubtless, ethyl ace toacetate.

A large number of experiments were made with the object of causing the hydrogen, which is produced during the synthesis of ethyl acetoacetate, to react with some readily reducible substance that was added to the ethyl acetate and sodium. The results of this work were not very decisive.

The experiments described above show, we think, that ethyl acetate, entirely free from alcohol, reacts with sodium as readily as do those 
specimens of the ester which probably contain at least a trace of alcohol. The addition of a small quantity of ether certainly does not lower the yield of ethyl acetoncetate. The velocity of the reaction is not re duced by the presence of ether.

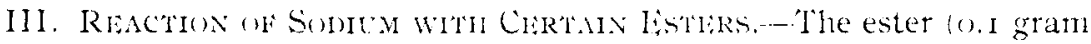
mol.) was mixed with "hexane" (200 cc, b. p. g( $\left.0^{\circ}\right)$ and heated with sodium wire.

Diethyl malonate, which was dissolved in petroleum ether (300 cc., b. p. $70^{\circ}$ ) instead of hexane, reacted quickly with the metal, hydrogen being evolved briskly at first. The mixture was boiled for about ist hours. A white powder formed which gradually turned yellow. Twogram atomic proportions of sodium reacted.

With diethyl chlormalonate the evolution of hydrogen was slight. Ifter five hours at the rom temperature the liquid began to turn yellow, and after 48 hours most of the wire had disappeared. After zo hours' boiling only one atomic proportion of sodium hacl reacted.

liethyl dimethylmalomatc, under sindilar conditions, reacted with one atomic proportion of sodium after two hours at the room temperature, the liquid becoming dark red. Five hours later a second atomic proportion of sodium had dissolved, and a third one after ten hours boiling. When the boiling was continued for about 70 hours longer the greater part of a fourth proportion of sodium reacted. The product consisted of a dark red solid which surrounded the metal and greatly hindered its contact with the remaining liquid. There was no appreciable evolution of hydrogen during this experiment. The bearing of these results on the constitution of the respective esters is discussed in the theoretical part of this paper.

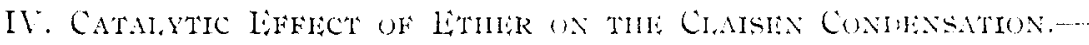
The following pairs of substances were condensed, in petroleum ether (b. p. $70^{\circ}$ ) solution, by means of sodinm wire: ethyl benzoate and acetone; ethyl oxalate and acetophenone: ethyl benzoate and acetophenone. Duplicate experiments were made in which petroleum ether containing 5 per cent. of absolute ether was employed. No differences could be observed either in the behavior of the materials or in the results of the experiments. In all cases the reactions proceeded very vigorously.

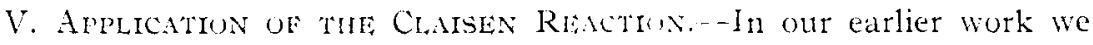
found that the best yield of the condensation product of camphor and ethyl benzoate was obtained by adding sodium ( 2 atoms) to the ester and treating the reaction product with sodium camphor, which contained a little sodium borneolate. We have applied this method to ethyl malunate, the solvent being hexane (b. p. $90^{\circ}$ ) containing a little ether. The vield of crude condensation product was about 50 per cent. of the ester employed. This process offers, therefore, no advantages over our earlier 
procedure, which consisted in adding sodium wire to the mixture of camphor, ester and solvent.

Ethyl phthalate, when treated with sodium (I atom), reacts immediately, but the wire soon becomes covered with a crust of white material which protects the metal from further attack. Consequently the yield of camphor condensation compound was small. These results show the necessity of studying carefully the behavior of each individual ester.

In consequence of our work with benzophenone and sodium, the results of which are described below, we attempted to condense this ketone with ethyl oxalate. The first experiments, with the ketone (I mol.), ester (I mol.) and sodium wire ( $\mathrm{r}$ atom), in petroleum ether (b. p. $36^{\circ}$ ), were not very successful, but on adding io per cent. of absolute ether to the petroleum ether, the reaction was rapid, the wire remained bright and all of it had reacted at the end of $i 2$ hours, at the ordinary temperature. The product was a dark brown solid, the solvent liquid being almost colorless. Similar results were obtained by the use of pyridine instead of ether. After extraction and acidification, in the manner described in our second paper, the condensation product was isolated. It consisted of a red oil, which, in alcoholic solution, gave an intense coloration with a solution of ferric chloride. Yield, 5o per cent. of the ketone. We desire to reserve the investigation of this compound; it may prove to be of considerable im. portance in connection with the study of the Claisen condensation.

The work described above shows that the catalytic influence of ether and the tertiary bases is quite general; the effect appears to be conditioned by the velocity with which the particular ketone and ester react. If this is relatively small the catalytic effect is great.

VI. Condensation of ACETAldehyde.-A very large number of experiments were made, under rather widely varied conditions, in order to obtain condensation products of acetaldehyde with ethyl oxalate, ethyl formate, amyl formate and camphor, respectively. The condensing agent employed was sodium, or the products formed by the action of this metal on camphor or one of the esters. In all cases the substances which were finally isolated appeared to be derived from the aldehyde alone. It will suffice to describe one experiment in which the aldehyde ( 2 mols.), dissolved in petroleum ether (b. p. $36^{\circ}$ ), was treated with sodium wire ( $\mathrm{I}$ atom). Reaction commenced immediately, and a white powder formed which darkened gradually. After about is minutes absolute ether, equal to 25 per cent. of the petroleum ether, was added. The mixture was allowed to remain for two hours and was then boiled during four hours. More acetaldehyde ( $1 \mathrm{~mol}$.) was now added and the heating continued for about 36 hours. The product was poured into water and the aqueous solution separated, acidified and extracted with ether. This ethereal solution contained a brown oil. It was treated first with potas- 
sium hydroxide, then with acid and gave a brilliant red powder, which gradually darkens in air. We have been mable to find a clescription of any such compound having been prepared previously from acetaldelycle.

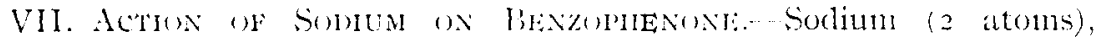
and benzophenone ( 1 mol.), in ether, react inmediately forming an intense blue powder. 'The action was practically complete after so hour's heating. Continued heating, in the presence of an additional atomic proportion of sodium, was without effect. The blue powder, which appears to be insoluble in ether, was treated with water, the ether removed (A), and the aqueous solution acidified and extracted with ether (B). From solution A we obtained benzhydrol (b. p. $297^{-208^{\circ}}$ ) and fron $B$ we isolated benzpinacone $\left(\mathrm{m}\right.$. p. I $\left.68^{\circ}\right)$. The rield of these purified products was 50 and 5.5 per cent., respectively, of the benzophenone. In a second experiment, similar to the above in all other respects, petroletm ether (b. p. $70^{\circ}$ ) was used as the solvent. No reaction took place at the room temperature until a little ether was added. The effect was then the same as in the previous case, but all the metal had disappearer after 5 hours' boiling. The blue powder was filtered quickly, washed with ether and then warmed with ether and metanitrobenzorl chloride. The product. when treated with water, gave a yellow crystalline precipitate which was purified by recrystallization from alcohol (95 per cent.). It melts above $300^{\circ}$. We hope to examine it more closely later.

These results show that benzophenone combines with two atomic proportions of sodium. In this case, too, ether appears to act catalytically in promoting the reaction.

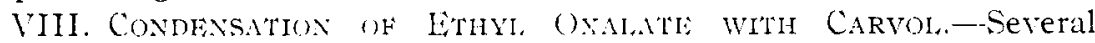
years ago Bishop Tingle and (y'Byme described some preliminary experiments ${ }^{1}$ on the condensation of ethyl oxalate with various phenols, in presence of sodium. They stated that carvol gave a resinous compound which had an odor of violets. At the end of last year Mr. F. M. Slocum informed the senior author that he had repeated this work several times and had been unable to obtain the odoriferous substance. We therefore carried out a number of experiments with carvol and ethyl oxalate, but we also failed to obtain any substance with the property in question. We employed carrol from Kahibaum and distilled it before use. Mr. Slocum's carvol was also highly purified. It appear's certain, therefore, that the fragrant smelling compound is not formed from carvol.

Bishop Tingle and O'Byrne's results were worked out with great care, under the strict supervision of the senior author, who has a distinct recollec tion of the powerful and unexpected odor of the substance in question. They used Kahlbaum's carvol as received, without further purification. In view of the discrepancy mentioned above and of the fact that, in the

${ }^{1}$ Am. Chem. J., 25, 50I (I)OI). 
paper cited, the description of the experiments is very brief, it may be well to give fuller details taken from the laboratory note-book. 'Carvol, (I 5 grams) was treated with ethyl oxalate (I I.2 grams), light petroleum ( $250 \mathrm{cc}$.) and sodium wire ( 2.3 grams). After the metal had dissolved the product was allowed to remain over-night and was then poured into water and the light petroleum solution removed. The aqueous portion of liquid was extracted with ether (A). The aqueous residue was then acidified and again extracted with ether (B). A and B were distilled and the residues, after being examined, were combined with that from the petroleum. This latter solution was also distilled, the temperature rising to $235^{\circ}$, up to which point two-thirds passed over. The residues were treated at the ordinary temperature with aqueous-alcoholic sodium hydroxide, the liquid was acidified, made alkaline with solution of sodium hydrogen carbonate and extracted twice with ether. The residue obtained after distilling this ethereal solution gave a deep red coloration with an alcoholic solution of ferric chloride. It was amorphous and had a strong fragrant odor of violets.'

\section{Summary.}

I. Ethyl acetate, highly purified by means of phosphoric anhydride and calcium, reacts with sodium as readily as that purified in the ordinary manner. Consequently the effect on the sodium cannot be due to traces of alcohol in the ester, as Nef and his students have stated.

2. Contrary to various statements which have been made, ethyl acetoacetate is formed without difficulty from sodium and ethyl acetate, in presence of ether.

3. Because of their varying behavior with sodium, diethyl malonate and diethyl dimethylmalonate appear to have different constitutions. Diethyl chlormalonate reacts as though it were intermediate between the other two.

4. The catalytic effect of ether and of tertiary bases in promoting the Claisen condentsation is only apparent if the velocity of the reaction is relatively small.

5. The Claisen reaction has been studied under various conditions and a condensation compound has been obtained from benzophenone and ethyl oxalate. The fact that benzophenone, which contains no $\mathrm{CH}_{3} \mathrm{CO}$ or $\mathrm{RCH}_{2} \mathrm{CO}$, reacts in this manner shows the entire error of the ClaisenNef hypothesis regarding the mechanism of the condensation.

6. Attempts to condense acetaldehyde with various esters were unsuccessful. The aldehyde formed complex polymerization or condensation products under the influence of the sodium.

7. Benzophenone combines with two atomic proportions of sodium. After treatment of the sodium derivative with water, the product consists chiefly of benzhydrol and benzpinacone. 
The experimental work clescribed in this paper was carried out by the junior author at the University of Rochester, $N$. Y., and at the Johns Hopkins University, during the session fgo 7 - i.

Mcmaster Univirity, Toronto, canain,

September, rgos.

\section{INTRAMOLECULAR REARRANGEMENT OF PHTHALAMIDIC ACIDS. III.}

BY J. BISHOP TINGLE AND H. F. ROLKER,

Received October $\pi$, ryos.

The previous papers ${ }^{1}$ on this subject described the formation of phthalphenylimide, $\mathrm{C}_{0} \mathrm{H}_{4} \backslash{ }_{\mathrm{CO}}^{\mathrm{CO}}>\mathrm{NC}_{6} \mathrm{H}_{5}$, from phthalphenylamidic (phthalanilic) acid, at a temperature about $100^{\circ}$ below its melting point, in presence of aniline. The fact that tertiary bases behave like aniline, led to the suggestion being made that the dehydration is due to salt formation. It was also shown that certain other phthalamidic acids behave in a similar manner, but not in an identical one. In the present communication we give an account of further work which we have done on this subject during the past year.

We have employed the following acids: phthalphenylamidic, $\mathrm{C}_{8} \mathrm{H}_{5} \mathrm{NHCOC}_{6} \mathrm{H}_{4} \mathrm{CO}_{2} \mathrm{H}$; phthalortho-, meta- and paratolylamidic, $\mathrm{CH}_{3} \mathrm{C}_{6} \mathrm{H}_{4} \mathrm{NHCOC}_{6} \mathrm{H}_{4} \mathrm{CO}_{2} \mathrm{H}$; phthalmeta- and paranitrophenylamidic, $\mathrm{O}_{2} \mathrm{NC}_{6} \mathrm{H}_{4} \mathrm{NHCOC}_{6} \mathrm{H}_{4} \mathrm{CO}_{2} \mathrm{H}$; and phthal- $\alpha-$ and $\beta$-naphthylamidic, $\mathrm{C}_{10} \mathrm{H}_{7} \mathrm{NHCOC}_{6} \mathrm{H}_{4} \mathrm{CO}_{2} \mathrm{H}$. The two nitro acids and the neta- and paratolyl acids have not been obtained previously.

As we required relatively large quantities of these acids we have worked out the details of their preparation and purification with care. Our method is described later in this paper (p. I885). It is very much simpler and more expeditious than the older process, but does not differ from it in general principles.

In the experiments recorcled in the earlier papers the acid and base were generally heated $2^{1 / 2}$ hours, or longer. In this later work we usually limited the time to 35 minutes because we wished to avoid the complete transformation of the acid into the imide. In this way we have been able to isolate certain intermediate products and have also succeeded, in some cases, in determining the relative velocity with which the various imides are formed.

We have investigated the effect of the following amines on the acids mentioned above: Pyridine, quinoline, ethylaniline, diphenylamine, aniline, $\alpha$-and $\beta$-naphthylamine and, in most cases, of benzylamine. In

${ }^{1}$ Bishop Tingle and Cram, Am. Chem. J., 37, 596 (1907); Bishop Tingle and Lovelace, Ibid., 38, 642 (1907). 